\title{
Demographics of the ALICE Collaboration
}

\author{
Anders G. Knospe $\mathbf{e}^{a, b, 1, *}$
}

${ }^{a}$ Department of Physics, Lehigh University

16 Memorial Drive East, Bethlehem, PA 18015, USA

${ }^{b}$ Department of Physics, University of Houston

3507 Cullen Blvd., Houston, TX 77204, USA

E-mail: anders.knospe@cern.ch

The ALICE Collaboration at the LHC is made up of around 1900 people from 39 countries. These proceedings discuss the composition of the Collaboration in terms of gender and career status. The distribution of responsibilities among various demographic groups will also be presented, and the time evolution of these statistics will be explored.

40th International Conference on High Energy physics - ICHEP2020

July 28 - August 6, 2020

Prague, Czech Republic (virtual meeting)

${ }^{1}$ For the ALICE Collaboration

*Speaker 


\section{Introduction}

The ALICE Diversity Office (ADO) was formed on 1 September 2019, following a recommendation from the ALICE Diversity Task Force. The Office consists of three members, appointed by the Spokesperson, with one recommended by the ALICE Juniors community (students and postdocs less than five years from their Ph.D.). The ADO has a mandate to advise collaboration members and management about diversity matters, promote diversity initiatives and maintain diversity web pages, monitor diversity issues and report periodically to the collaboration, and liaise with diversity offices at CERN and in other LHC experiments. We meet periodically with the diversity offices of the other LHC experiments, including conversations facilitated by the CERN Diversity and Inclusion Programme. We are in the process of organizing training sessions on inclusive leadership, which will be offered to Physics Working Group conveners and others holding leadership positions within ALICE. In summer 2020, we conducted a survey on diversity-related matters, following up an earlier survey conducted by the Diversity Task force. The results of this survey will be released in 2021. We maintain open lines of communication with the membership of the ALICE Collaboration, both via email and via an anonymous comment box on our website [1]. We invite any ALICE Collaboration member with questions, comments, or concerns to contact us; confidentiality is guaranteed.

In addition, the ALICE Diversity Office analyzes data relating to diversity, inclusion, and the demographics of the ALICE Collaboration. This effort complements the aforementioned survey and is the topic of these proceedings.

\section{Methodology}

The data presented in these proceedings come from three sources. First is the GLANCE database. This contains detailed information about every ALICE Collaboration member, past and present, starting in 2014. Information includes name, dates of membership, employing institute, career status, and various positions of responsibility held within the ALICE Collaboration (including dates). Also included is information on gender, which is unfortunately limited to binary female/male options. (In the survey mentioned above, we included additional gender options to cover persons with non-binary gender identities, or who preferred not to disclose their genders.) Members were grouped into multiple employment categories: Early Students (summer, bachelor, masters), Ph.D. Students, Postdocs, Senior Scientists (those with indefinite or permanent positions), Engineers/Technicians, and "Other" (which includes administrative staff, honorary members, guests, and additional roles). Members were also grouped into geographic regions based on the location of their employing institute. However, we are in the process of changing the geographic divisions, so these results will not be presented in these proceedings. A "snapshot" of the collaboration database was taken for 1 September of each year, establishing a person's employment and geographic category for that year. The number of collaboration responsibilities held on 1 September of a given year was also tabulated. Such responsibilities include collaboration leaders, Physics Working Group conveners, detector project leaders, members of a variety of boards and committees, and team (institute) leaders. 
These data from the GLANCE database were correlated with information provided by the ALICE Editorial Board (which manages collaboration publications) and the ALICE Conference Committee (which oversees collaboration contributions to various conferences, including talks, posters, and proceedings). For each ALICE publication, two committees are formed. The Paper Committee (PC) consists of the people who worked on the analysis in question, as well as other ALICE members with relevant expertise on the subject. The PC is responsible for writing the paper on behalf of the collaboration. The Internal Review Committee (IRC), also unique for each paper, is responsible for reviewing the work of the PC and serves as a group of referees within the collaboration. The Editorial Board supplied a list of all ALICE publications, including the members of the PC and IRC, the dates these committees were formed, and the date the paper was released to the public (taken to be the end of the tenures of the two committees). From these data, the ADO tabulated the number of PC and IRC assignments held by each collaboration member on 1 September of each year. The ALICE Conference committee supplied a list of all presentations given on behalf of ALICE in each year. The ADO tabulated the number of presentations given by each member of the collaboration in each year. Presentations were divided into multiple categories so that we could analyze separately the distribution of more prominent presentations. In order of prominence, these categories are Posters at all Conferences, Talks at all Conferences, Talks at Minor Conferences, Talks at Major Conferences, and Plenary Talks at Major Conferences. Note that some categories are subsets of others. Major conferences are defined as European Physical Society, Hard Probes, Initial Stages, ICHEP, LHCP, Quark Matter, and Strangeness in Quark Matter. The data on publications and presentations were analyzed together with the basic demographic information from the GLANCE database with the goal of seeing whether demographic characteristics affect the assignment of responsibilities within the collaboration.

\section{Results and Discussion}

A selection of results from this study (excluding geographic information) will now be presented and discussed.

The total membership of the ALICE Collaboration was 1,550 in 2014 and rose to 1,901 in 2019. The fraction of members who are women is about 20\%, rising slightly from $18.3 \%$ in 2014 to $20.1 \%$ in 2019. Fig. 1 (left) shows the number of people in each employment category, divided by gender. Fig. 1 (right) shows the fraction of people within each employment category who are women and the faction who are men. Women make up about $30 \%$ of the Early Student category, which is above the collaboration average and the average for the Ph.D. Student and Postdoc categories. The remaining categories, including Senior Scientists and Engineers/Technicians have fewer women than the collaboration average. This indicates that there is a net loss of women from the ALICE Collaboration as the career stage advances.

Fig. 2 (left) shows the fraction of women making up each employment category as a function of time. A large increase in the fraction of women is seen for the Early Students category, with a smaller increase for Ph.D. students. However, consistently increasing trends are not observed for the fraction of women in other employment categories. Given the increasing trends for students, we are hopeful that similar increases might become apparent for other employment categories in the coming years. 

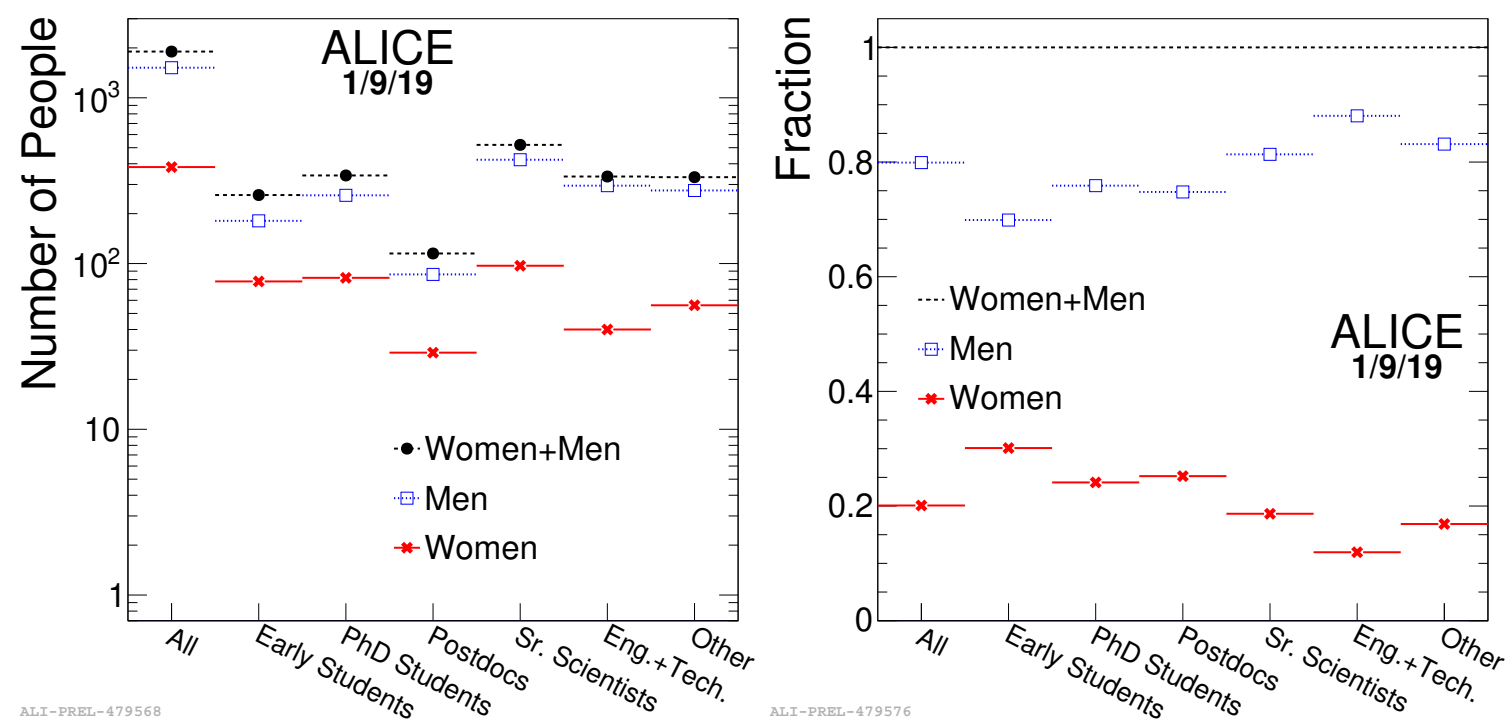

Figure 1: Left: Number of people in each employment category. Right: Fraction of people in each employment category by gender. (Both panels: snapshot for 1 September 2019.)
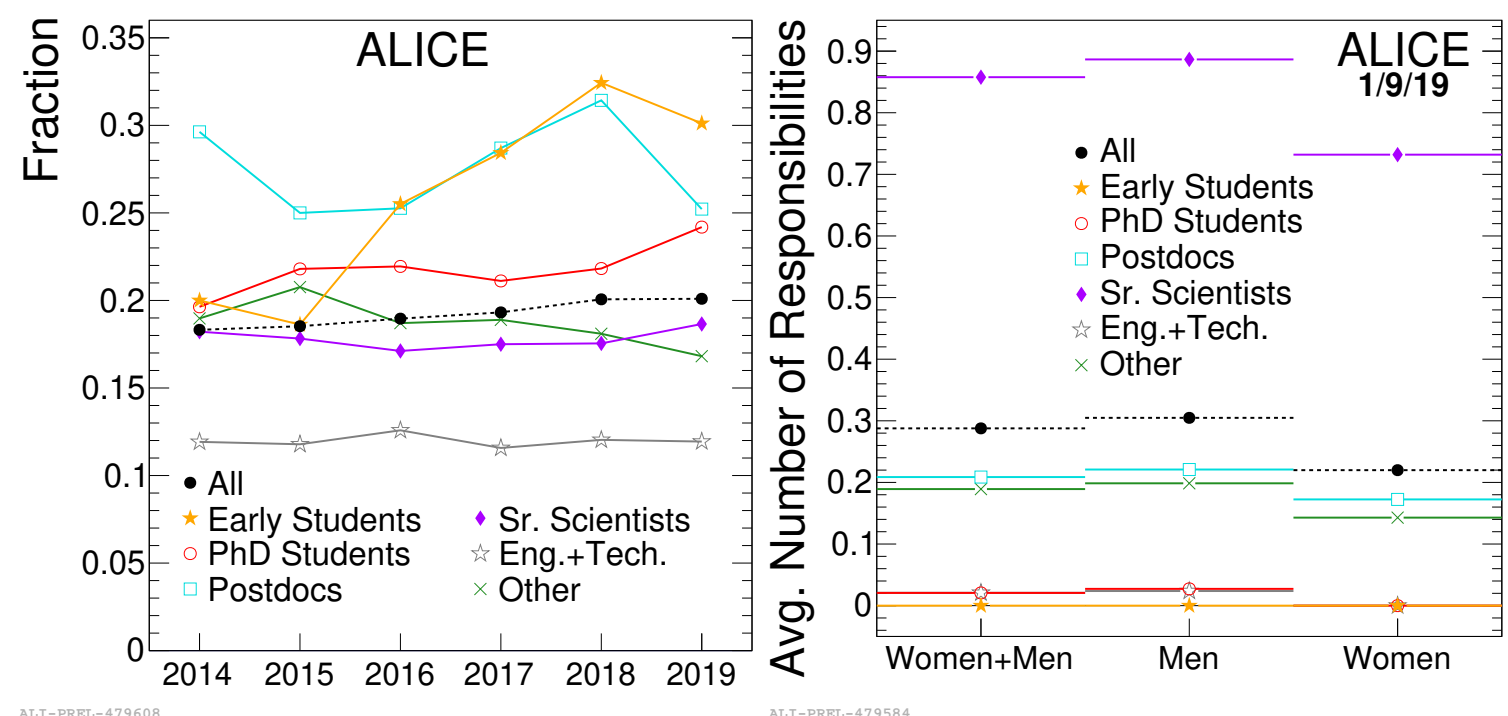

Figure 2: Left: Fraction of women making up each employment category vs. time (snapshot each year for 1 September). Right: Average number of responsibilities held by collaboration members, divided by gender and employment category (snapshot for 1 September 2019). 

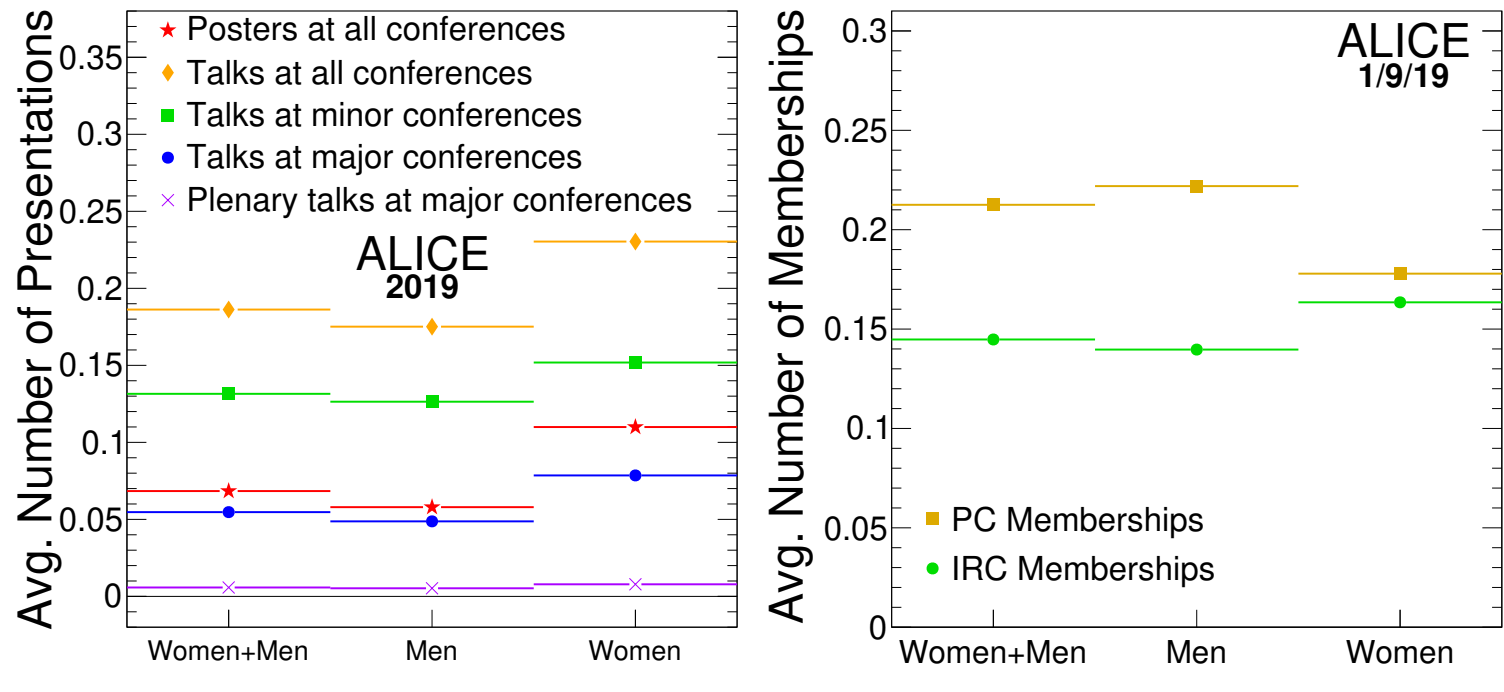

Figure 3: Left: Average number of presentations given throughout 2019 at various types of conferences, divided by gender. Right: Average number of Paper Committee (PC, writes the paper) and Internal Review Committee (IRC, reviews the paper) memberships held, divided by gender (snapshot for 1 September 2019).

The average number of responsibilities held by members of the collaboration, divided by gender and employment category, are shown in Fig. 2 (right). PC and IRC memberships are studied separately and are not included in the totals here. As expected, the number of responsibilities increases with career level. It is also noted that women tend to hold fewer responsibilities than men, a trend which persists for all employment categories.

Fig. 3 (left) shows the average number of presentations given by members of the collaboration, divided by gender and type of presentation. On average, women give more presentations than men, which is true for all types of presentations. For reference, twelve presentations (three given by women) were classified as Plenary Talks at Major Conferences for 2019.

Fig. 3 (right) shows the average numbers of PC and IRC memberships held by members of the collaboration on 1 September 2019, divided by gender. On average, women hold below the average number of Paper Committee memberships, but above the average number of Internal Review Committee memberships.

As shown in the presentation at the conference itself, we have also performed similar studies for different geographic regions, based on the location of a person's employing institution. We identified cases in which people from some geographic regions held below the average number of responsibilities or gave fewer presentations at conferences. (The interpretation of how presentations are distributed by region is complicated ay an additional factor: conferences themselves are not necessarily distributed evenly by geographic region, and people may be more likely to attend conferences that are in near their own region.) However, due to our pending redefinition of the geographic regions within ALICE, we will not report geographic studies in these proceedings. 


\section{Conclusions}

As part of its mandate, ALICE Diversity Office conducts a regular study of the demographics of the ALICE Collaboration and the assignment of responsibilities and presentations to collaboration members. We observe that women make up about $20 \%$ of the collaboration, a fraction that has increased slightly with time from 2014 to 2019 . We identified some areas that may warrant special attention from the leadership of the collaboration, including the number of responsibilities held by women. We also identified a few geographic regions where collaboration members tend to hold fewer responsibilities and/or give fewer talks. We observe some positive trends in these data, but note that certain areas still require attention. We are assembling a full report on this study, including new definitions of geographic regions. These results, along with the results of our recent survey, will be released in the coming months.

\section{Acknowledgments}

Thanks to the other members of the ALICE Diversity Office, past and present: Adriana Telesca, Ejiro Naomi Umaka, and Fernando Flor. Together, we thank the leadership of the ALICE Collaboration for their support and cooperation. We thank the ALICE Editorial Board and Conference Committee for supplying the necessary data. The members of the ALICE Diversity Office thank CERN, the University of Houston, and Lehigh University for their support of our work in this office. We thank the CERN Diversity and Inclusion Programme and the other LHC diversity offices for fruitful discussions; we look forward to continuing our collaborations with you.

\section{References}

[1] https://alice-collaboration.web.cern.ch/Diversity0ffice 